\title{
Radio spectrum evolution and magnetic field in extreme GPS radio sources The case of RXJ1459+3337
}

\author{
M. Orienti ${ }^{1,2,3}$ and D. Dallacasa ${ }^{2,3}$ \\ 1 Instituto de Astrofisica de Canarias, 38200 La Laguna, Tenerife, Spain \\ e-mail: orienti@ira.inaf.it \\ 2 Dipartimento di Astronomia, Università di Bologna, via Ranzani 1, 40127 Bologna, Italy \\ 3 Istituto di Radioastronomia - INAF, via Gobetti 101, 40129 Bologna, Italy
}

Received 15 June 2007 / Accepted 5 November 2007

ABSTRACT

\begin{abstract}
Aims. The knowledge of the properties of the youngest radio sources is very important in order to trace the earliest phase of the evolution of the radio emission. RXJ1459+3337, with its high turnover frequency $(\sim 25 \mathrm{GHz})$ provides a unique opportunity to study this class of extreme objects.

Methods. High-sensitivity multi-frequency VLA observations have been carried out to measure the flux-density with high accuracy, while multi-frequency VLBA observations were performed, aimed at determining the pc-scale structure. Archival ROSAT data have been used to infer the X-ray luminosity.

Results. The comparison between our new VLA data and those available in the literature shows a steady increment of the flux-density in the optically-thick part of the spectrum and a decrement of the turnover frequency. In the optically-thin regime, the source flux density has already started to decrease. Such a variability can be explained in terms of an adiabatically-expanding homogeneous radio component. The frequency range spanned by our VLBA observations, together with the resolution achieved, allows us to determine the source size and the turnover frequency, and then to derive the magnetic field directly from these observable quantities. The value obtained in this way is in good agreement with that computed assuming equipartition condition. A similar value is also obtained by comparing the radio and X-ray luminosities.
\end{abstract}

Key words. galaxies: active - galaxies: evolution - radio continuum: general - magnetic fields - radiation mechanisms: non-thermal

\section{Introduction}

The radio emission of extragalactic sources is synchrotron radiation produced by relativistic electrons with a power-law energy distribution. The relativistic electrons produce a power-law radio spectrum which reaches its maximum in correspondence to the turnover frequency $v_{t}$.

At frequencies below $v_{t}$, the spectrum turns over likely due to synchrotron self-absorption (Snellen et al. 2000), although freefree absorption may also play a role (Mutoh et al. 2002; Kameno et al. 2000).

The evolutionary models proposed to interpret the various stages of the life-cycle of radio sources relate the typical spectral peak of very small and then compact region with age. In the evolutionary scenario, the peak of the spectrum progressively moves toward lower frequencies as the radio source expands/grows. In this framework, GHz-peaked spectrum (GPS) and compact steep-spectrum (CSS) radio sources, the former with $v_{t} \sim 1 \mathrm{GHz}$ and the latter with $v_{t} \sim 100-500 \mathrm{MHz}$, are considered to represent early stages in the individual radio source evolution.

The timescale of the spectral evolution in extremely young radio sources is very short, of the order of a few tens of years (Dallacasa 2003). This implies that the ideal targets to investigate how the radio spectrum evolves, and that are the main mechanisms at work, must be sought among sources with very high turnover frequencies. The existence of "extreme" GPS sources with turnover frequency above $10 \mathrm{GHz}$, termed "high frequency peakers" (HFPs) by Dallacasa et al. (2000), is expected from all the radio source evolutionary models. Their detection is, however, quite difficult since they are short-lived objects evolving into GPS and then CSS sources, and to observational limitations preventing their selection. For a source to be recognized as an extreme GPS object, the characteristic turnover frequency must lie within the frequency range sampled by large area surveys. The frequencies of the surveys currently available are not high enough to allow an efficient selection of high-frequency peaking objects, since there is insufficient information above $\sim 5 \mathrm{GHz}$.

The radio source RXJ1459+3337 was one of the first objects to be recognized as an "extreme" GPS with the turnover frequency at about $30 \mathrm{GHz}$ and a peak flux density of about $800 \mathrm{mJy}$ as reported by Edge et al. (1996). This object is associated with a quasar at $z=0.6448$. It was detected by ROSAT (Brinkmann et al. 2000) and shows an X-ray luminosity $L_{\mathrm{X}} \sim 2.8 \times 10^{45} \mathrm{erg} / \mathrm{s}$.

Multi-epoch VLA observations indicate strong variability in the optically-thick part of its radio spectrum. In particular, the flux-density variability at $5 \mathrm{GHz}$ (i.e. in the optically-thick regime) seems to steadily increase. Although flux-density variability is quite rare in GPS sources (O'Dea 1998), an increment of the flux density at frequencies below $v_{t}$ may be a consequence of the evolution of a synchrotron self-absorbed spectrum in an expanding component. 
In this paper we present the results of multi-frequency VLA and VLBA observations conducted in 2003 and 2005, respectively. In order to identify the mechanisms at the basis of the spectral evolution we combine the information on the physical conditions provided by our new VLA data with that from archival data obtained in 1996 and 1999. The frequency range sampled by the new VLBA observations, together with their high-resolution, allows us to compute the magnetic field by means of observable quantities only, such as peak frequency, peak flux density and the source size, as from the synchrotron theory (Kellermann \& Pauliny-Toth 1981; see also Sect. 4.2). The comparison between this value with the field strength obtained assuming equipartition provides important information on the physical conditions of the radio source.

Throughout this paper, we assume the following cosmology: $H_{0}=71 \mathrm{~km} \mathrm{~s}^{-1} \mathrm{Mpc}^{-1}, \Omega_{\mathrm{M}}=0.27$ and $\Omega_{\lambda}=0.73$, in a flat Universe.

\section{Observations and data reduction}

Our target RXJ1459+3337 was observed with the VLA on September 12th 2003 at 8 independent frequencies $(1.4,1.7,4.5$, $4.9,8.1,8.4,15.0$ and $22.2 \mathrm{GHz}$ ) during the monitoring program of HFP candidates (Dallacasa et al. 2000; Tinti et al. 2005), the characteristics of the observations and the data reduction are described in Orienti et al. (2007). In VLA data the rms noise level on the image plane is not relevant (always well below $1 \mathrm{mJy}$ ), if compared to the main uncertainty coming from the amplitude calibration errors, which are within $(1 \sigma) 3 \%$ at $1.4,1.7,4.5,5.0$, 8.1 and $8.4 \mathrm{GHz}$, and $5 \%$ at 15 and $22 \mathrm{GHz}$, and which are predominant in the case of relatively strong radio sources such as RXJ1459+3337.

VLBA observations of the radio source RXJ1459+3337 were carried out on April 4th 2005 at 1.7, 5, 8.4, 15, 22 and $43 \mathrm{GHz}$, in full polarization mode with a recording bandwidth of $32 \mathrm{MHz}$ at $128 \mathrm{Mbps}$, for a total time of 10 hours. The correlation was performed at the VLBA correlator in Socorro and the data reduction was carried out with the NRAO AIPS package. After the application of system temperature and antenna gain, the amplitudes were checked using the data on 4 C39.25 (J0927+3902). The error on the absolute flux density scale is generally within $3 \%-10 \%$, being worse at the highest frequencies. The same source 4C 39.25 was used to generate the bandpass correction at each frequency. However, at $43.2 \mathrm{GHz}$ a problem on the scan on the calibrator precluded a good bandpass calibration.

Images of RXJ1459+3337 at each frequency were produced after a number of phase-only self-calibration iterations (Fig. 1). The source was found to be marginally resolved at all frequencies (Fig. 2). The source flux densities at each frequency have been measured on both VLBA and VLA images in several ways, giving similar results, and the values are reported in Table 1. The VLBA flux-densities at each frequency are consistent within $<10 \%$ with those measured by the VLA, indicating the lack of low-surface brightness features on the pc-scale.

\section{Results}

GPS radio sources are usually characterized by the lack of any significant flux-density variability and they can be considered as the least variable class of extragalactic radio sources (O'Dea 1998), with an average variation within $~ 5 \%$ (Stanghellini et al. 2005). In the case of RXJ1459+3337, however, a comparison between our new simultaneous multi-frequency VLA observations with those available from the literature has pinpointed a substantial variability in the optically thick part of the spectrum.

Figure 3 shows the light-curves of RXJ1459+3337 at each frequency: from these plots it seems that at 1.4 and $5 \mathrm{GHz}$, well below the turnover frequency, the flux density has steadily increased, while in the optically-thin regime (i.e. $22 \mathrm{GHz}$ ), the flux density has decreased. Following the approach from Dallacasa et al. (2000), we fit the simultaneous radio spectra with a purely analytical function used to determine the peak flux density $S_{m}$ and the frequency $v_{m}$ at which it occurs:

$\log S=a-\sqrt{b^{2}+(c \log (v)-d)^{2}}$.

In this equation, the parameters $a, b, c$ and $d$ are purely numeric, and do not provide any direct physical information. Based on the results of the fit, we found that the spectral peak has moved to lower frequencies, from $\sim 24 \pm 1 \mathrm{GHz}$ in 1996 to $17 \pm 2 \mathrm{GHz}$ in 1999 and to $12.5 \pm 0.5$ in 2003 (Fig. 4). The errors on these quantities were calculated following the error propagation theory.

The decrement of the spectral peak together with the steady increment of the flux-density in the optically-thick part of spectrum suggest that the source is adiabatically expanding. Despite the high resolution achieved by VLBA observations, the radio source RXJ1459+3337 is only marginally resolved even at $43 \mathrm{GHz}$. Since the inferred source size is substantially smaller than the VLBA beam size at the highest frequency, our measurements are not sensitive to small changes on the angular size and thus cannot confirm the source expansion suggested by the spectrum evolution.

\section{Discussion}

The strong variability in the optically thick part of the spectrum is not common among sources peaking below $5 \mathrm{GHz}$ (O’Dea 1998). However, the timescale of evolution of extreme GPS sources appears to be quite short, of the order of a few tens of years. In the context of the youth scenario, such a result is not unexpected since very young radio sources should evolve quite rapidly (Dallacasa 2003).

In the following discussion we investigate the physical conditions of the radio emission and which mechanisms are involved in the evolution of the radio spectrum.

\subsection{The evolution of the radio spectrum}

As noted above, the radio emission in extragalactic radio sources is due to synchrotron radiation from relativistic particles with a power-law energy distribution. However, in very small objects the observed radio spectrum significantly departs from the power-law shape, turning over at frequencies below the peak. Such a deviation is mostly explained in terms of synchrotron self-absorption.

The mechanism which plays the major role in the evolution of the optically-thick part of the spectrum is the adiabatic expansion. In this regime we have that

$S(v) \propto H^{-\frac{1}{2}} v^{\frac{5}{2}} \theta^{2}$

(Kellermann \& Pauliny-Toth 1981) where $S$ is the flux density at a given frequency $v$ in the optically-thick part of the spectrum, $H$ is the magnetic field and $\theta$ is the angular size of the emitting region. As the radio source adiabatically expands, the opacity 

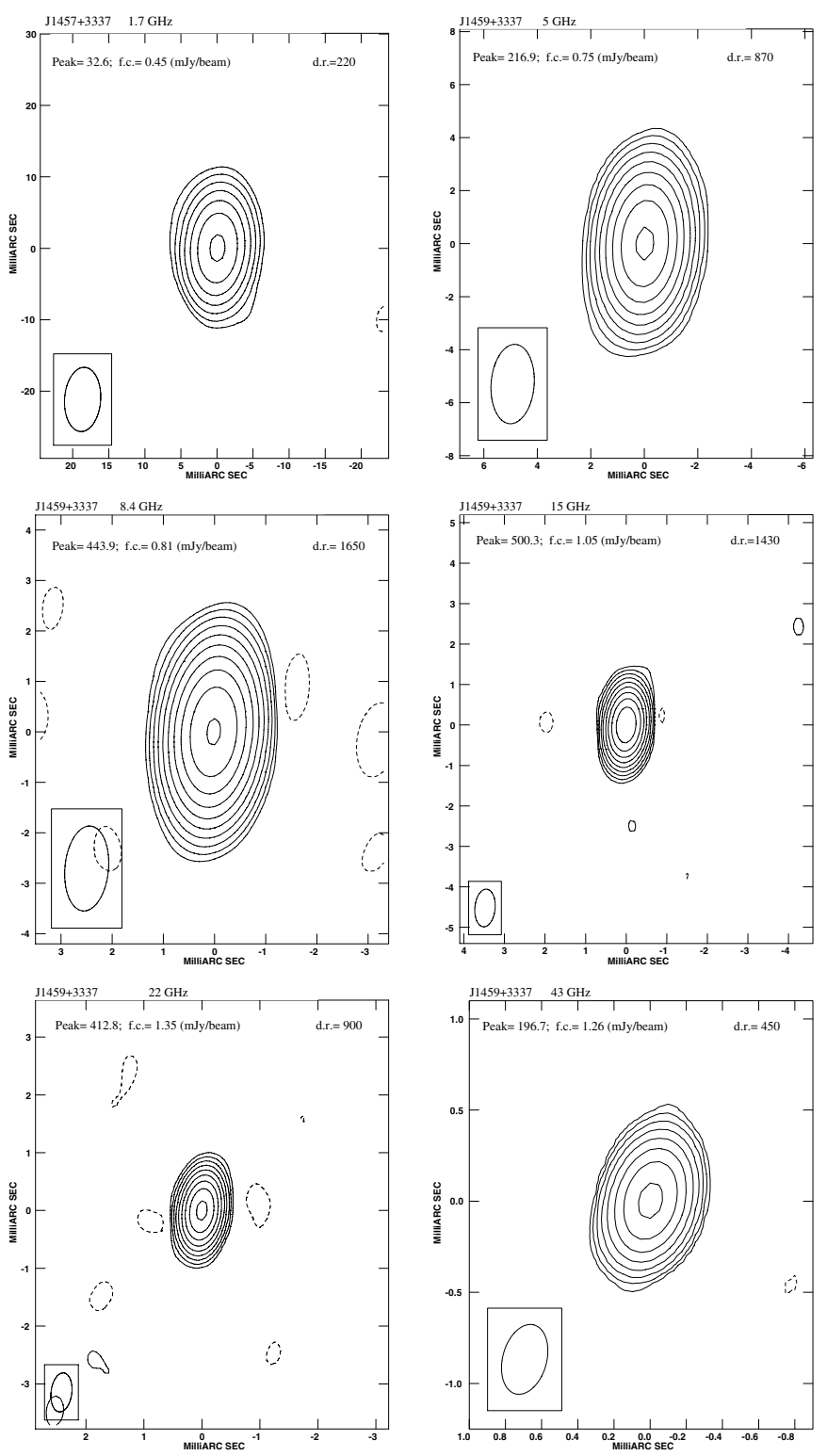

Fig. 1. VLBA images of the source J1459+3337 at 1.7, 5, 8.4, 15, 22 and $43 \mathrm{GHz}$. On each image we give the following information on the plot itself: a) peak flux density in $\mathrm{mJy} / \mathrm{beam}$; b) first contour intensity ( $\mathrm{mJy} /$ beam), which is generally equal to $3 \mathrm{rms}$ of the noise level; contour levels increase by a factor of 2 ; c) the dynamic range (the ratio of the peak brightness and $1 \sigma$ ); d) the restoring beam, plotted on the bottom left corner of each image.

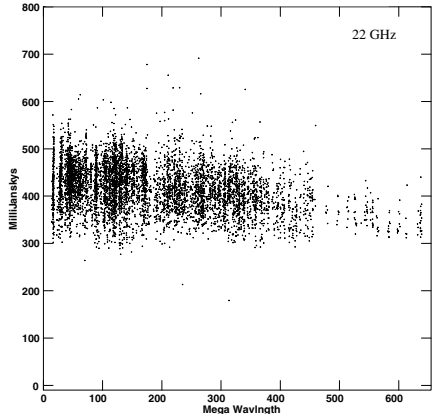

Fig. 2. Plot of amplitude vs. projected baseline length at $22 \mathrm{GHz}$.

decreases, the turnover frequency moves to lower frequencies and the flux density increases at frequencies below the turnover frequency.
Is it possible to relate the flux-density variability observed in RXJ1459+3337 to adiabatic expansions?

First of all, we assume that the radio emission is due to a homogeneous component which is adiabatically expanding at a constant rate:

$\theta=\theta_{0}\left(\frac{t_{0}+\Delta t}{t_{0}}\right)$

where $\theta_{0}$ is the source angular size at the time $t_{0}$ and $\theta$ at the time $t_{0}+\Delta t$.

We also assume that the magnetic field is frozen in the plasma:

$H=H_{0}\left(\frac{t_{0}}{t_{0}+\Delta t}\right)^{2}$ 
Table 1. The VLA and VLBA flux densities of RXJ1459+3337.

\begin{tabular}{ccccccccccc}
\hline \hline & Date & $\begin{array}{c}S_{1.4} \\
\mathrm{mJy}\end{array}$ & $\begin{array}{c}S_{1.7} \\
\mathrm{mJy}\end{array}$ & $\begin{array}{c}S_{4.5} \\
\mathrm{mJy}\end{array}$ & $\begin{array}{l}S_{5.0} \\
\mathrm{mJy}\end{array}$ & $\begin{array}{l}S_{8.1} \\
\mathrm{mJy}\end{array}$ & $\begin{array}{c}S_{8.4} \\
\mathrm{mJy}\end{array}$ & $\begin{array}{c}S_{15} \\
\mathrm{mJy}\end{array}$ & $\begin{array}{c}S_{22} \\
\mathrm{mJy}\end{array}$ & $\begin{array}{c}S_{43} \\
\mathrm{mJy}\end{array}$ \\
\hline VLA & Sep. 2003 & 25 & 31 & 194 & 221 & 410 & 423 & 521 & 356 & \\
VLBA & Apr. 2005 & & 33 & & 221 & & 446 & 519 & 348 & 198 \\
\hline
\end{tabular}
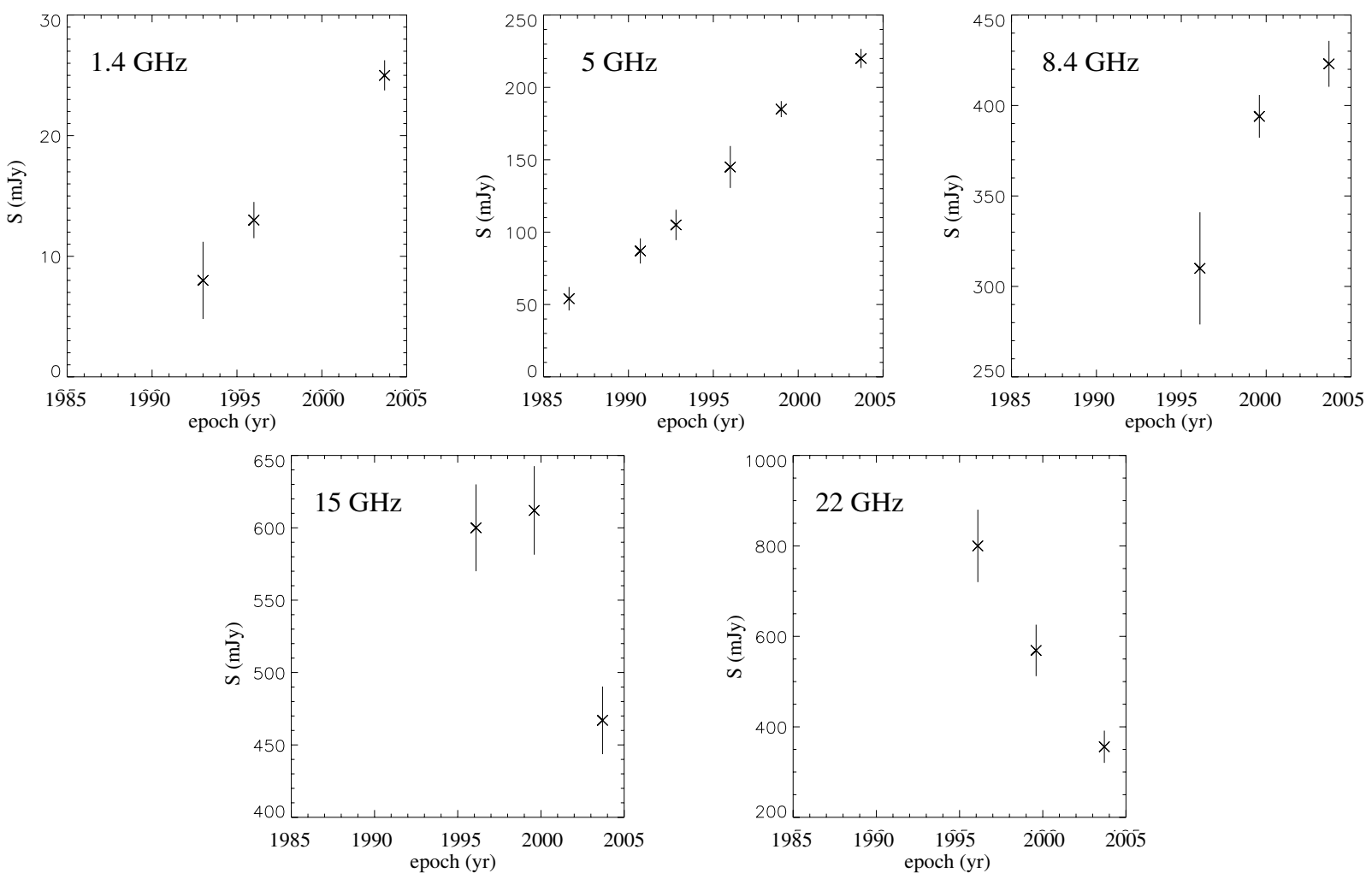

Fig. 3. Light-curves of RXJ1459+3337 at 1.4, 5, 8.4, 15 and $22 \mathrm{GHz}$. In addition to the data points presented in this paper we include those concerning the epochs 1986 (87GB, Gregory et al. 1996), 1991 (Neumann et al. 1994), 1993 (Laurent-Muehleisen et al. 1997 ), 1996 (Edge et al. 1996) and 1999 (Dallacasa 2003).

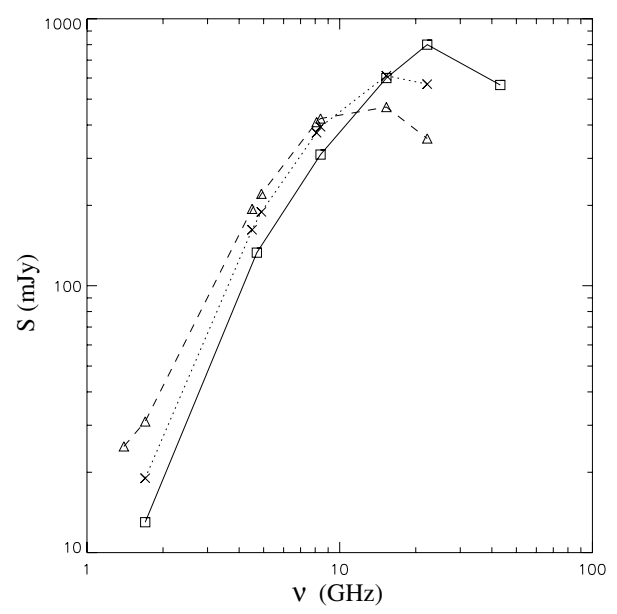

Fig. 4. The VLA radio spectrum of RXJ1459+3337 in 1996 (squares, Edge et al. 1996), in 1999 (crosses, Dallacasa 2003), and in 2003 (triangles, these new data).

where $H_{0}$ is the magnetic field at the time $t_{0}$ and $H$ the magnetic field at the time $t_{0}+\Delta t$. From Eq. (1) we find that:

$\frac{S}{S_{0}}=\left(\frac{t_{0}+\Delta t}{t_{0}}\right)^{3}$ where $S_{0}$ and $S$ are the flux densities measured at the same frequency at the time $t_{0}$ and $t_{0}+\Delta t$ respectively.

In the case of the $5 \mathrm{GHz}$ light-curve, where enough data points are available, we estimate the time of onset of radio emission by fitting the flux density measured at the different epochs with the function

$y=b+(x / a)$

where $y=\left(S / S_{0}\right)^{1 / 3}, x=t-t_{0}, a$ the age the radio emission had at epoch $t_{0}$ and $b$ a free parameter which is almost equal to unity in our fits. As $S_{0}$ we consider the flux density measured at the oldest epoch available $\left(t_{0}\right.$; i.e. 1986). From the best fit to the data we obtain $a \sim 30 \pm 5$ years, i.e. the birth of the radio emission should have occurred in $1956 \pm 5$. In the case of the $1.4 \mathrm{GHz}$ light-curve, the availability of only 3 data points makes the determination of the age less accurate with $a \sim 27 \pm 15$ years, i.e. the radio emission should have started in $1966 \pm 15$, in agreement with the $5 \mathrm{GHz}$ determination. We do not perform fits on the light-curves at higher frequencies since they are affected by contamination due to the flattening occurring near the spectral peak.

As previously mentioned, adiabatic expansion also causes the spectral peak to shift at lower frequencies. We know that the turnover frequency is

$v_{t} \propto E^{2} H$ 
where the energy of the relativistic particles is defined as $E \propto t^{-1}$ (Pacholczyk 1970). This implies that

$\frac{v_{0}}{v}=\left(\frac{t_{0}+\Delta t}{t_{0}}\right)^{4}$

where $v_{0}$ and $v$ are the turnover frequency at the time $t_{0}$ and $t_{0}+\Delta t$ respectively.

We estimate the epoch of the origin of radio emission by fitting the frequency peak measured at the different epochs with the same function used to fit the multi-epoch flux density. In this case, $y=\left(v_{0} / v\right)^{1 / 4}, x=t-t_{0}$ and $a$ the age the radio emission had at the epoch $t_{0} . v_{0}$ is the peak frequency at the oldest epoch available ( $t_{0}$, in this case 1996). From the best fit we find $a \sim 47 \pm 10$ years, implying that the radio emission originated at $\sim 1949 \pm 10$, still in agreement with the values obtained from the flux density increment, although with higher uncertainties, since the fit has been performed on 3 data points only. The good agreement on the determination of the source age may indicate that the radio source is evolving with the physical conditions predicted by simple self-similar growth models, as previously assumed.

\subsection{The magnetic field}

Direct measurements of the magnetic field are very difficult to carry out. The magnetic field $H$ can be determined from observable quantities, such as the turnover frequency $v_{t}$ in $\mathrm{GHz}$, the peak flux density $S_{\text {max }}$ in Jy and the source angular sizes $\theta_{\text {maj }}$ and $\theta_{\min }$ in mas as directly inferred from the observations. In this case we have

$H \sim 10^{-4} \theta_{\text {maj }}^{2} \theta_{\text {min }}^{2} v_{t}^{5} S_{\text {max }}^{-2}(1+z)^{-1}$

(Kellermann \& Pauliny-Toth 1981) where $H$ is in Gauss. From the fit to our new VLA data (see Sect. 2), we obtained $v_{t} \sim$ $12.5 \pm 0.5 \mathrm{GHz}$ and $S_{\max } \sim 550 \pm 15 \mathrm{mJy}$. In addition, by using the task JMFIT we measured a source angular size of $0.36 \times 0.14$ mas $\left(\theta=1.8 \times \theta_{\text {fit }}\right.$ where $\theta_{\text {fit }}$ is the deconvolved full width at half-maximum of a Gaussian brightness distribution as used in the fitting procedure) on our best VLBA image, namely that at $22 \mathrm{GHz}$, since the longest spacings at $43 \mathrm{GHz}$ are poorly sampled and with a rather high noise. The resulting resolution at $43 \mathrm{GHz}$ is therefore no better than that at $22 \mathrm{GHz}$. With these parameters we obtain $H \sim 0.16 \pm 0.03 \mathrm{G}$.

Another way to constrain the magnetic field is to assume that the radio source is in equipartition conditions. From the minimum energy conditions (Pacholczyk 1970), the equipartition magnetic field is

$H=\sqrt{\frac{24}{7} \pi u_{\min }}$

where the minimum energy density $u_{\min }$ is

$u_{\min }=7 \times 10^{-24} \cdot\left(\frac{L}{V}\right)^{4 / 7} \cdot(1+k)^{4 / 7}$

and $L$ is the luminosity calculated at $22 \mathrm{GHz}$, i.e. in the opticallythin part of the spectrum, and $V$ the volume of the radio source in $\mathrm{pc}^{3}$. For the calculation of $V$, we assume that the radio emission has an ellipsoidal geometry and an average optically thin spectral index of $0.7\left(S \propto v^{-\alpha}\right)$. Furthermore, proton and electron energies are assumed to be equal $(k=1)$, with a filling factor of unity (i.e. the source volume is fully and homogeneously filled by relativistic plasma). From Eq. (6), we calculate an equipartition magnetic field $H_{\mathrm{eq}} \sim 0.16 \mathrm{G}$, in very good agreement with the value directly derived from the observed spectral quantities.

Since RXJ1459+3337 is also an X-ray source, we can try to constrain the magnetic field by comparing the radio and X-ray luminosities, if we assume that all the X-ray emission is due to Comptonization of the electrons responsible for the synchrotron emission in the radio band. In this case we have:

\section{$\frac{L_{\mathrm{SSC}}}{L_{\mathrm{Syn}}} \sim \frac{8 \pi w_{f}}{H^{2}}$}

(e.g. Singal 1986) where $L_{\text {Syn }}$ and $L_{\mathrm{SSC}}$ are the synchrotron and the synchrotron self-Compton luminosity respectively and $w_{f}$ is the radiation field, defined as

$w_{f}=\frac{3 L_{\text {Syn }}}{A c}$

with $A$ the surface area of the synchrotron emission, and $c$ the speed of light. The ROSAT X-ray flux density of RXJ1459+3337 is $1.56 \times 10^{-12} \mathrm{erg} \mathrm{s}^{-1} \mathrm{~cm}^{-2}$ (Brinkmann et al. 2000 ), which corresponds to an X-ray luminosity $L_{\mathrm{SSC}} \sim 2.8 \times$ $10^{45} \mathrm{erg} / \mathrm{s}$. From the radio flux density we derive a synchrotron luminosity $L_{\text {Syn }} \sim 1.2 \times 10^{45} \mathrm{erg} / \mathrm{s}$.

If in Eq. (7) we introduce these luminosities and the source emitting area $A \sim 3.9 \mathrm{pc}^{2}$ as derived from VLBA images assuming an ellipsoidal geometry (see above), we obtain a magnetic field $H \sim 0.19 \mathrm{G}$, still in good agreement with the values derived by the previous methods.

These results strongly suggest that the radio emitting plasma in such an extreme object is consistent with the equipartition conditions.

\section{Conclusions}

We have presented new multi-frequency VLBA and VLA observations of the radio source RXJ1459+3337. Given the position of the spectral peak at very high frequency, this object is classified as an extreme GPS radio source, also known as HFP.

By comparing our VLA observations with data from the literature spanning about 17 years, we find that the flux-density in the optically-thick part of the spectrum has been continuously increasing, while the turnover frequency has been moving toward lower frequencies. Such a regular variability, although not common in GPS sources peaking below $5 \mathrm{GHz}$, can be described in the case the radio emission originates from an adiabaticallyexpanding homogeneous component.

If in this context we compare either the flux-densities at the same frequency measured at different times or the turnover frequencies, we constrain the age of the radio emission, which is about $\sim 50 \pm 10$ years.

The resolution achieved by the VLBA observations, together with the frequency range sampled, allowed us to "observe" the turnover frequency and to directly measure parameters, such as the peak flux density and angular size of the source with great accuracy. In this way we estimate the magnetic field to be $0.16 \pm 0.03 \mathrm{G}$ by means of observable quantities only. The consistency of this value with the field derived for a minimum energy condition strongly supports the idea that such extreme objects, as RXJ1459+3337 may already be in equipartition. The availability of ROSAT X-ray observations allowed us to infer the magnetic field by comparing radio and X-ray luminosities. Even in this case, the value inferred for the magnetic field agrees with the previous results. 
Acknowledgements. We thank the anonymous referee for carefully reading the manuscript and valuable suggestions. The VLA and VLBA are operated by the US National Radio Astronomy Observatory which is a facility of the National Science Foundation operated under a cooperative agreement by Associated University, Inc. This work has made use of the NASA/IPAC Extragalactic Database NED which is operated by the JPL, California Institute of Technology, under contract with the National Aeronautics and Space Administration.

\section{References}

Becker, R. H., White, R. L., \& Helfand, D. J. 1995, ApJ, 450, 559

Brinkmann, W., Laurent-Muehleisen, S. H., Voges, W., et al. 2000, A\&A, 356, 445

Dallacasa, D. 2003, PASA, 20, 79

Dallacasa, D., Stanghellini, C., Centonza, M., \& Fanti, R. 2000, A\&A, 363, 887

Edge, A. C., Jones, M., Saunders, R., Pooley, G., \& Grainge, K. 1996, in Proceedings of the Second Workshop on GPS and CSS Radio Sources, ed. I. A. G. Snellen et al. (Leiden: Leiden Observatory), 208
Gregory, P. C., Scott, W. K., Douglas, K., \& Condon, J. J. 1996, ApJS, 103, 427 Kameno, S., Horiuchi, S., Shen, Z.-Q., et al. 2000, PASJ, 52, 209

Kellermann, K. I., \& Pauliny-Toth, I. I. K. 1981, ARA\&A, 19, 373

Laurent-Muehleisen, S. A., Kollgaard, R. I., Ryan, P. J., et al. 1997, A\&AS, 122, 235

Mutoh, M., Makoto, I., Kameno, S., et al. 2002, PASJ, 54, 131

Neumann, M., Reich, W., Fuerst, E., et al. 1994, A\&AS, 106, 303 O'Dea, C. P. 1998, PASP, 110, 493

Orienti, M., Dallacasa, D., \& Stanghellini, C. 2007, A\&A, 475, 813

Pacholkczyk, A. G. 1970, Radio Astrophysics (San Francisco: Freeman \& Co.) Polatidis, A. G., \& Conway, J. E. 2003, PASA, 20, 69

Singal, A. K. 1986, A\&A, 155, 242

Snellen, I. A. G., Schilizzi, R. T., Miley, G. K., et al. 2000, MNRAS, 319, 445

Stanghellini, C., O'Dea, C. P., Dallacasa, D., et al. 2005, A\&A, 443, 891

Tinti, S., Dallacasa, D., de Zotti, G., Celotti, A., \& Stanghellini, C. 2005, A\&A, 432, 31 